\title{
Electron Time of Transit Spectroscopy for Analysis and Imaging
}

\author{
David C Joy \\ University of Tennessee and Oak Ridge National Laboratory, Oak Ridge TN37831 \\ Joe Goldstein's numerous and significant contributions to electron microscopy have \\ enhanced both microanalysis and imaging. In that context it is therefore appropriate to \\ take a look at a concept - time of transit electron spectroscopy - which could bring new \\ capabilities to both of these areas when applied in a scanning electron microscope.
}

The interaction of energetic electrons with a solid is usually treated as being an instantaneous event. In reality, the time taken between the entry of the electron into the solid and the emergence of a backscattered or secondary electron is finite, a fact which has several consequences. For example, the magnitude of this 'transit' time potentially sets an upper limit to the scan speed of an SEM since the raster should not advance to the next pixel position until emission has ceased from the previous pixel. The transit time also determines the maximum response speed, and noise characteristics, of devices such as photomulitplier tubes and electron multipliers. Finally, a knowledge of time intervals associated with electron transport could facilitate the application of a 'time of flight' spectroscopy for electrons as a means for controlling the spatial resolution of imaging and as a method of materials analysis. These phenomena can be modeled by a Monte Carlo trajectory tracing method since the position and the velocity of every electron is known at all times. In the work described here the interval between the entry of the incident electron and the exit of backscattered electrons (BSE) has been determined for a variety of elements and as a function of the incident electron energy. The program also stores the radial distance between the entrance and exit positions of the BSE so that exit positions can be correlated with transit times. For a beam energy of $10 \mathrm{keV}$ the average time between electron entry and subsequent backscattering varies from 20 femptoseconds $\left(2 * 10^{-14}\right.$ secs $)$ for carbon to about 2 fempto-seconds $\left(2 * 10^{-15} \mathrm{secs}\right)$ for gold. The transit time varies as a function of the velocity of the incident electron, so that at $30 \mathrm{keV}$ the corresponding times for carbon and gold would be 80 f.secs and 8 f.secs respectively. As a result, and rather counter-intuitively, higher energy electrons and electrons traveling in a low atomic number low density material take a longer time to leave than less energetic electrons in a denser material. At $10 \mathrm{keV}$ the maximum time for the emergence of a BSE is of the order of $35 \mathrm{f}$.secs for carbon and $10 \mathrm{f}$.secs for gold, suggesting that 100 f.secs is probably a lower safe limit for imaging pixel times.

Plots of the number of BSE emerging from a sample after a given time have a profile which varies systematically with the atomic number of the target, as shown in figure (1), as well as showing significant differences in the time scale of the interaction. This suggests that if the transit time could be determined with a precision in the femptosecond range then, although not elementally specific, this time of flight method might provide a route to ultra-high speed 'triage' analysis of materials particularly since the yield of BSE events is very high ( $\sim 0.2$ /incident electron $)$ compared to that of X-rays or Auger electrons $\left(\sim 10^{-6} /\right.$ incident electron). 
An examination of the exit positions and the exit time shows (figure 2) that there is no unique correlation, although typically $90 \%$ of the BSE signal collected in a given time window comes from a relatively narrow region within the interaction volume. This provides the ability to control the spatial resolution of the backscattered image although at the expense of signal to noise ratio. Initial results gained from extending this study to secondary electron (SE) emission show that the temporal characteristics are quite similar in form because the exit time of an SE is closely related to that of the incident (for SE1 production) or exiting backscattered electron (for SE2 production) that generates it.

\section{References}

(1) Research sponsored by the Assistant Secretary for Energy Efficiency and Renewable Energy, Office of FreedomCAR and Vehicle Technology Program, as part of the High Temperature Materials Laboratory User Program, Oak Ridge National Laboratory managed by UT-Batelle, LLC for U.S. Department of Energy under contract number DE-AC05-00OR22725

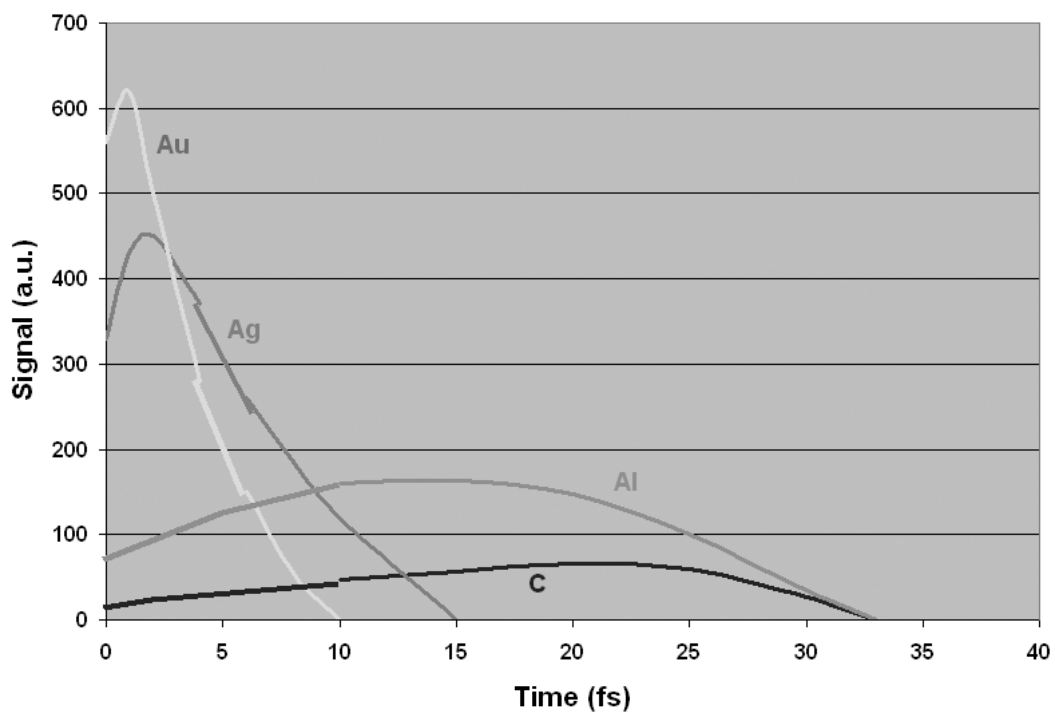

Figure 1. Exit time spectra for BSE generated by $10 \mathrm{keV}$ beam incidence on carbon, aluminum, silver and gold. The spectra were computed for the same number of incident electrons so the signal intensity is a measure of the backscattering coefficient

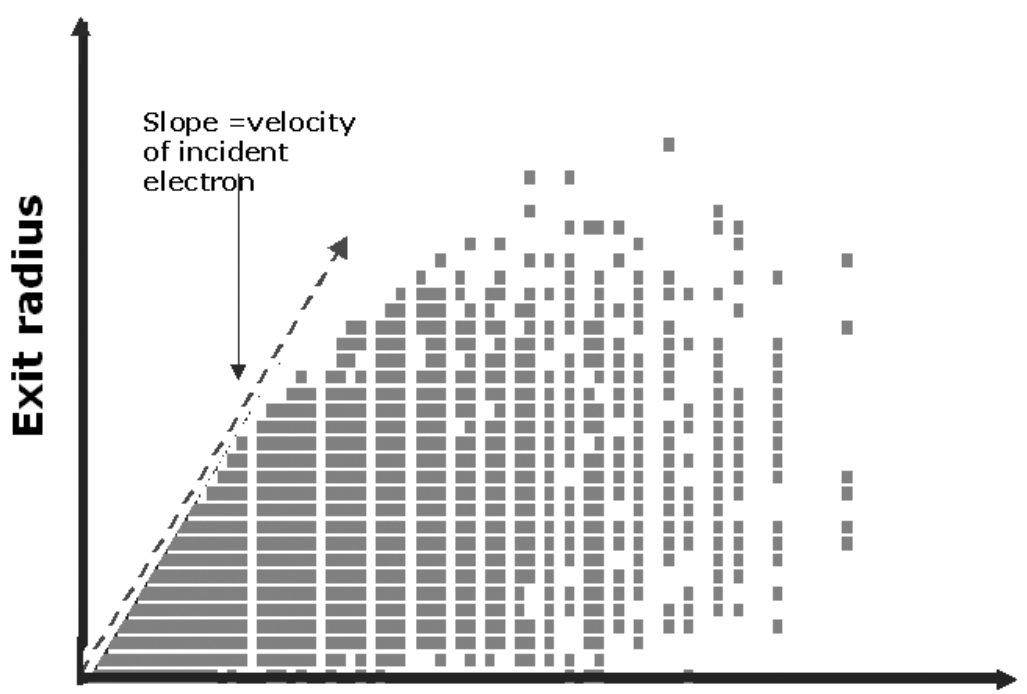

Figure 2. Plot of BSE exit radius versus exit time for $10 \mathrm{keV}$ incident electrons on silicon. The upper bound of the distribution is the velocity of the incident electron. The banding is an artifact of the plotting routine. 